\title{
Irreversibility and the Arrow of Time in a Quenched Quantum System
}

\author{
T. B. Batalhão, ${ }^{1,2}$ A. M. Souza, ${ }^{3}$ R. S. Sarthour, ${ }^{3}$ I. S. Oliveira,${ }^{3}$ M. Paternostro, ${ }^{4}$ E. Lutz, ${ }^{5}$ and R. M. Serra ${ }^{1,6}$ \\ ${ }^{1}$ Centro de Ciências Naturais e Humanas, Universidade Federal do ABC, Avenida dos Estados 5001, \\ 09210-580 Santo André, São Paulo, Brazil \\ ${ }^{2}$ Faculty of Physics, University of Vienna, Boltzmangasse 5, Vienna A-1090, Austria \\ ${ }^{3}$ Centro Brasileiro de Pesquisas Físicas, Rua Dr. Xavier Sigaud 150, 22290-180 Rio de Janeiro, Rio de Janeiro, Brazil \\ ${ }^{4}$ Centre for Theoretical Atomic, Molecular and Optical Physics, School of Mathematics and Physics, Queen's University, \\ Belfast BT7 1NN, United Kingdom \\ ${ }^{5}$ Department of Physics, Friedrich-Alexander-Universität Erlangen-Nürnberg, 91058 Erlangen, Germany \\ ${ }^{6}$ Department of Physics, University of York, York YO10 5DD, United Kingdom
}

(Received 15 June 2015; revised manuscript received 2 September 2015; published 2 November 2015)

Irreversibility is one of the most intriguing concepts in physics. While microscopic physical laws are perfectly reversible, macroscopic average behavior has a preferred direction of time. According to the second law of thermodynamics, this arrow of time is associated with a positive mean entropy production. Using a nuclear magnetic resonance setup, we measure the nonequilibrium entropy produced in an isolated spin-1/2 system following fast quenches of an external magnetic field. We experimentally demonstrate that it is equal to the entropic distance, expressed by the Kullback-Leibler divergence, between a microscopic process and its time reversal. Our result addresses the concept of irreversibility from a microscopic quantum standpoint.

The microscopic laws of classical and quantum mechanics are time symmetric, and hence reversible. However, paradoxically, macroscopic phenomena are not time-reversal invariant $[1,2]$. This fundamental asymmetry defines a preferred direction of time that is characterized by a mean entropy production. Regardless of the details and nature of the evolution at hand, such entropy production is bound to be positive by the second law of thermodynamics [3]. Since its introduction by Eddington in 1927 [4], the thermodynamic arrow of time has not been tested experimentally at the level of a quantum system.

Introduced by Clausius in the form of an uncompensated heat, the importance of the entropy production in nonequilibrium statistical physics was recognized by Onsager and further developed by Meixner, de Groot, and Prigogine [5]. Defined as $\Sigma=\beta(W-\Delta F)$, for a system at constant inverse temperature $\beta=1 /\left(k_{B} T\right)$, where $W$ is the total work done on the system and $\Delta F$ the free energy difference ( $k_{B}$ is the Boltzmann constant), it plays an essential role in the evaluation of the efficiency of thermal machines, from molecular motors to car engines [3].

Starting with Boltzmann's work on the so-called $H$ theorem, the quest for a general microscopic expression for the entropy production, especially far from equilibrium, has been a challenge for more than a century [1]. In the last years, formulas for the entropy production and entropy production rate in terms of the microscopic density operator $\rho$ of a system have been obtained for relaxation [6], transport [7], and driven processes in closed and open quantum systems [8,9]. At the same time, the recent development of fluctuation theorems $[10,11]$ has led to a sharpening of the formulation of the second law. Regardless of the size of a system, the arrow of time originates from the combination of an explicit time dependence of the Hamiltonian of the system and the specific choice of an initial equilibrium state. While the first ingredient breaks time homogeneity (thus inducing the emergence of an arrow of time), the second specifies its direction [12].

In small systems, thermal and quantum fluctuations are both significant, and fluctuation theorems quantify the occurrence of negative entropy production events during individual processes [13]. In particular, the average entropy production $\langle\Sigma\rangle$ for evolution in a time window $\tau$ has been related to the Kullback-Leibler relative entropy between states $\rho_{t}^{F}$ and $\rho_{\tau-t}^{B}$ along the forward and backward (i.e., time reversed) dynamics [14-16] (see Fig. 1). Explicitly,

$$
\langle\Sigma\rangle=S\left(\rho_{t}^{F} \| \rho_{\tau-t}^{B}\right)=\operatorname{tr}\left[\rho_{t}^{F}\left(\ln \rho_{t}^{F}-\ln \rho_{\tau-t}^{B}\right)\right] .
$$

The above equation quantifies irreversibility at the microscopic quantum level and for the most general dynamical process responsible for the evolution of a driven closed system. A process is thus reversible, $\langle\Sigma\rangle=0$, if forward and backward microscopic dynamics are indistinguishable. Nonequilibrium entropy production and its fluctuations have been measured in various classical systems, ranging from biomolecules [17] and colloidal particles [18] to levitated nanoparticles [19] (see Refs. [20,21] for a review). Evidence of time asymmetry has been further observed in a driven classical Brownian particle and its electrical 


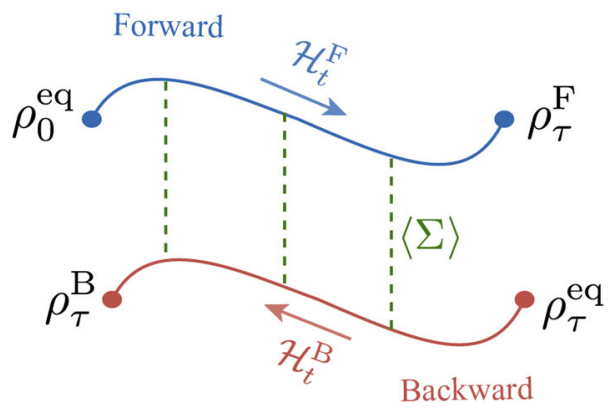

FIG. 1 (color online). A quantum system (with Hamiltonian $\mathcal{H}_{0}^{F}$ ) is initially prepared in a thermal state $\rho_{0}^{\mathrm{eq}}$ at inverse temperature $\beta$. It is driven by a fast quench into the nonequilibrium state $\rho_{\tau}^{F}$ along a forward protocol described by the Hamiltonian $\mathcal{H}_{t}^{F}$. In the backward process, the system starts in the equilibrium state $\rho_{\tau}^{\text {eq }}$ corresponding to the final Hamiltonian $\mathcal{H}_{\tau}^{F}$ and is driven by the time-reversed Hamiltonian $\mathcal{H}_{t}^{B}=\mathcal{H}_{\tau-t}^{F}$ to $\rho_{\tau}^{B}$. The entropy production $\langle\Sigma\rangle$ at time $t$ is given by the KullbackLeibler divergence between forward and backward states $\rho_{t}^{F}$ and $\rho_{\tau-t}^{B}$ [cf. Eq. (1)].

counterpart [22]. However, quantum experiments have remained elusive so far, owing to the difficulty to measure thermodynamic quantities in the quantum regime. To date, Eq. (1) has thus never been tested.

In this Letter, we use a nuclear magnetic resonance (NMR) setup to provide a clear-cut assessment of Eq. (1) where $\langle\Sigma\rangle$ and $S\left(\rho_{t}^{F} \| \rho_{\tau-t}^{B}\right)$ are tested and evaluated independently. Our methodological approach is founded on the reconstruction of the statistics of work and entropy, following a nonequilibrium process implemented on a twolevel system, therefore assessing the emergence of irreversibility (and the associated arrow of time) starting from a genuine microscopic scale.

We consider a liquid-state sample of chloroform, and encode our system in the qubit embodied by the nuclear spin of ${ }^{13} \mathrm{C}$ [23-25]. The sample is placed in the presence of a longitudinal static magnetic field (whose direction is taken to be along the positive $z$ axis) with strong intensity, $B_{0} \approx 11.75 \mathrm{~T}$. The ${ }^{13} \mathrm{C}$ nuclear spin precesses around $B_{0}$ with Larmor frequency $\omega_{\mathrm{C}} / 2 \pi \approx 125 \mathrm{MHz}$. We control the system magnetization through rf-field pulses in the transverse ( $x$ and $y$ ) directions [25]. The initial thermal state $\rho_{0}^{\mathrm{eq}}$ of the ${ }^{13} \mathrm{C}$ nuclear spin (at inverse temperature $\beta$ ) is prepared by suitable sequences of transversal radiofrequency (rf)-field and longitudinal field-gradient pulses. We use the value $k_{B} T / h=1.56 \pm 0.07 \mathrm{kHz}$ (corresponding to an effective temperature of $T \simeq 75 \pm 3 \mathrm{nK}$ ) throughout the experiment for the initial ${ }^{13} \mathrm{C}$ nuclear spin thermal states. A sketch of the experimental setup is provided in Fig. 2.

The system is driven out of equilibrium to the state $\rho_{\tau}^{F}$ by a fast quench of its Hamiltonian (denoted as $\mathcal{H}_{t}^{F}$ in this forward process) lasting a time $\tau$. We experimentally realize

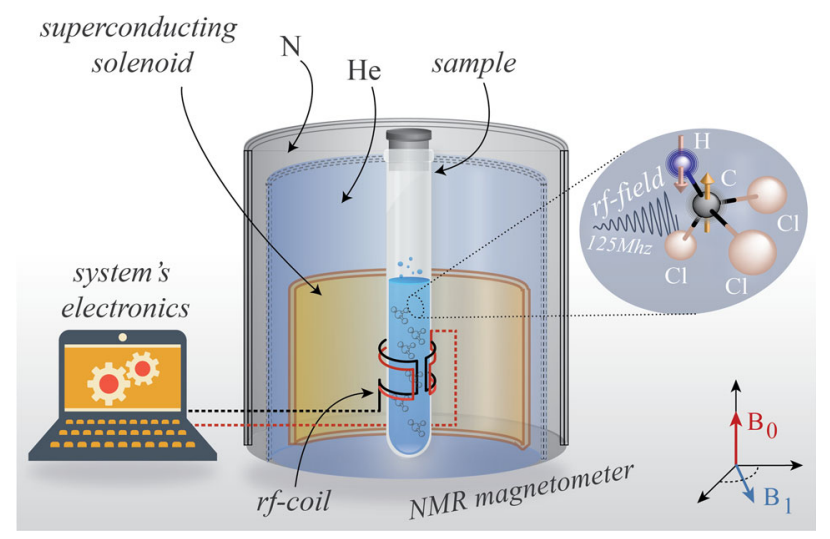

FIG. 2 (color online). We show a section of the magnetometer employed in our NMR experiment. A superconducting magnet, which produces a high intensity magnetic field $\left(B_{0}\right)$ in the longitudinal direction, is immersed in liquid $\mathrm{He}$, surrounded by liquid $\mathrm{N}$ in another vacuum separated chamber, in a thermally shielded vessel. The liquid sample (inside a $5 \mathrm{~mm}$ glass tube) is placed at the center of the magnet within the rf coil of the probe head. A digital electronic time-modulated pulse induces a transverse rf field $\left(B_{1}\right)$ that drives the ${ }^{13} \mathrm{C}$ nuclear spins out of equilibrium. In the forward (backward) protocol, the rf coil can perform (retrieve) work on (produced by) the nuclear spin sample. Depending on the speed of the modulation of the driving field, irreversible entropy is produced. The sketch is not in scale and has been stripped of unnecessary technical details of the apparatus.

this quench by a transverse time-modulated rf field set at the frequency of the nuclear spin. In a rotating frame at the spin Larmor frequency, the Hamiltonian regulating the forward process is

$$
\mathcal{H}_{t}^{F}=2 \pi \hbar \nu(t)\left[\sigma_{x}^{\mathrm{C}} \cos \phi(t)+\sigma_{y}^{\mathrm{C}} \sin \phi(t)\right]
$$

with $\sigma_{x, y, z}^{\mathrm{C}}$ the Pauli spin operators, $\phi(t)=\pi t /(2 \tau)$, and $\nu(t)=\nu_{0}(1-t / \tau)+\nu_{\tau} t / \tau$ the (linear) modulation of the rf-field frequency over time $\tau$, from the value $\nu_{0}=1.0 \mathrm{kHz}$ to $\nu_{\tau}=1.8 \mathrm{kHz}$. With these definitions, the initial thermal state of the ${ }^{13} \mathrm{C}$ system is $\rho_{0}^{\mathrm{eq}}=\exp \left(-\beta \mathcal{H}_{0}^{F}\right) / Z_{0}$, where $Z_{0}$ is the partition function at time $t=0$.

In order to reconstruct the work and entropy production statistics of the ${ }^{13} \mathrm{C}$ quenched dynamics, we use the method proposed in Refs. [26-28] and illustrated in detail in Ref. [29]. We consider an ancillary qubit embodied by the ${ }^{1} \mathrm{H}$ nuclear spin of our sample (Larmor frequency $\omega_{\mathrm{H}} / 2 \pi \approx 500 \mathrm{MHz}$ ) and exploit the natural scalar coupling between the ${ }^{1} \mathrm{H}$ and ${ }^{13} \mathrm{C}$ nuclear spins to implement the interferometer needed to reconstruct the statistics of the work done by ${ }^{13} \mathrm{C}$ following the quench [29]. The method assumes a unitary dynamics of both system and ancilla, a condition that is met with excellent accuracy in our experiment. In fact, the spin-lattice relaxation times, measured by the inversion recovery pulse sequence, are 
$\left(\mathcal{T}_{1}^{\mathrm{H}}, \mathcal{T}_{1}^{\mathrm{C}}\right) \approx(7.36,10.55) \mathrm{s}$. The transverse relaxations, obtained by the Carr-Purcell-Meiboom-Gill pulse sequence, have characteristic times $\left(\mathcal{T}_{2}^{\mathrm{H}}, \mathcal{T}_{2}^{\mathrm{C}}\right) \approx$ $(4.76,0.33) \mathrm{s}$. We thus study processes of maximal duration $\tau \sim 10^{-4} \mathrm{~s}$ and consider total data acquisition times for the reconstruction of the work and entropy statistics within 0.1 and $126 \mathrm{~ms}$, being smaller than $\mathcal{T}_{1}^{\mathrm{H}, \mathrm{C}}$. This enables us to disregard any energy exchange with the system environment during the quenched dynamics. The effects of the ${ }^{13} \mathrm{C}$ transverse relaxation $\left(\mathcal{T}_{2}^{\mathrm{C}}\right)$ can be partially overcome by a refocussing strategy in the reconstruction procedure.

We implement the backward process, shown in Fig. 1, by driving the system with the time-reversed Hamiltonian $\mathcal{H}_{t}^{B}=\mathcal{H}_{\tau-t}^{F}$ from the equilibrium state $\rho_{\tau}^{\mathrm{eq}}=$ $\exp \left(-\beta \mathcal{H}_{\tau}^{F}\right) / Z_{\tau}$ that corresponds to the final Hamiltonian $\mathcal{H}_{\tau}^{F}\left(Z_{t}\right.$ here denotes the partition function at time $\left.t\right)$. The intermediate ${ }^{13} \mathrm{C}$ states during the field quench are $\rho_{t}^{F}=$ $\mathcal{U}_{t} \rho_{0}^{\mathrm{eq}} \mathcal{U}_{t}^{\dagger}$ and $\rho_{\tau-t}^{B}=\mathcal{V}_{\tau-t} \rho_{\tau}^{\mathrm{eq}} \mathcal{V}_{\tau-t}^{\dagger}$, where the evolution operators satisfy the time-dependent Schrödinger equations $d_{t} \mathcal{U}_{t}=-i \mathcal{H}_{t}^{F} \mathcal{U}_{t}$ and $d_{t} \mathcal{V}_{t}=i \mathcal{H}_{\tau-t}^{F} \mathcal{V}_{t}$ with the initial conditions $\mathcal{U}_{0}=\mathcal{V}_{0}=\mathbb{1}$.

Work is performed on the system during the forward and backward processes. The corresponding probability distributions $P^{F, B}(W)$ are related via the Tasaki-Crooks fluctuation relation [30-32]

$$
P^{F}(W) / P^{B}(-W)=e^{\beta(W-\Delta F)} .
$$

Equation (3) characterizes the positive and negative fluctuations of the quantum work $W$ along single realizations. It holds for any driving protocol, even beyond the linear response regime, and is a generalization of the second law to which it reduces on average, $\langle\Sigma\rangle=\beta(\langle W\rangle-\Delta F) \geq 0$.

We experimentally verify the arrow of time expressed by Eq. (1) by determining both sides of the equation independently. We first evaluate the Kullback-Leibler relative entropy between forward and backward dynamics by tracking the state of the spin $1 / 2$ at any time $t$ with the help of quantum state tomography [23]. Figures 3(a)-3(c) show reconstructed trajectories followed by the Bloch vector, for both forward and backward processes and different quench times. As a second step, we measure the probability distribution $P(\Sigma)$ of the irreversible entropy production using the Tasaki-Crooks relation (3). Employing NMR spectroscopy [23] and the method described in Refs. [26-28] (cf. Ref. [29] for a detailed analysis), we determine the forward and backward work distributions, from which we extract $\beta, W$, and $\Delta F$, and hence the entropy produced during each process. The measured nonequilibrium entropy distribution is shown in Fig. 4(a). It is discrete as expected for a quantum system. We further observe that both positive and negative values occur owing to the stochastic nature of the problem. However, the mean entropy production is positive (red (a)

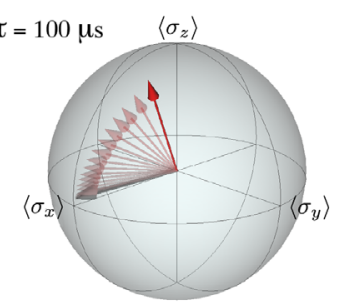

(b)

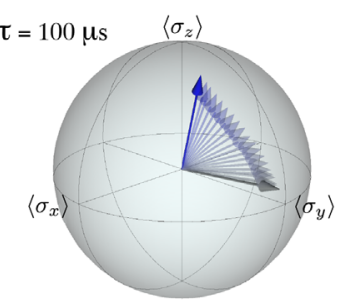

(c) $\tau=100 \mu \mathrm{s}$

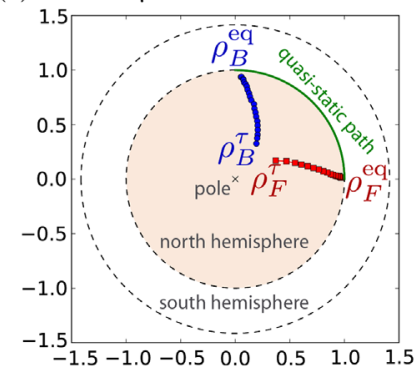

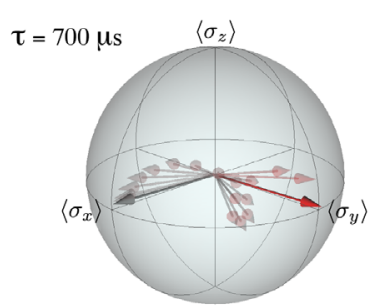
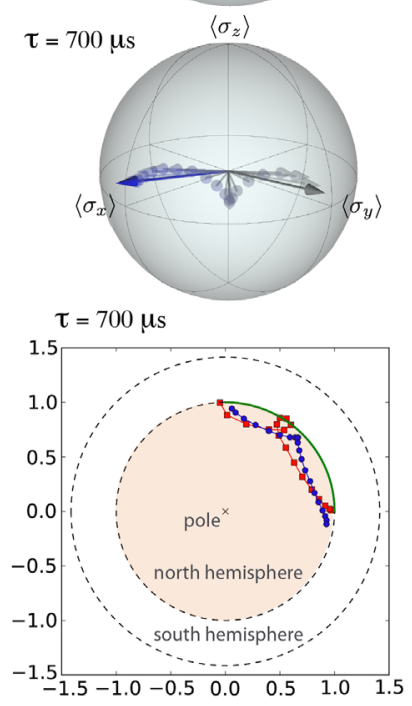

FIG. 3 (color online). (a),(b) Evolution of the Bloch vector of the forward [backward] spin- $1 / 2$ state $\rho_{t}^{F}\left[\rho_{\tau-t}^{B}\right]$ during a quench of the transverse magnetic field, obtained via quantum state tomography. A sampling of 21 intermediate steps has been used. The initial magnetization (gray arrow) is parallel to the external rf field, aligned along the positive $x[y]$ axis for the forward [backward] process. The final state is represented as a red [blue] arrow. (c) Polar projection (indicating only the magnetization direction) of the Bloch sphere with the trajectories of the spin. Green lines represent the path followed in a quasistatic $(\tau \rightarrow \infty)$ process.

line) in agreement with the Clausius inequality $\langle\Sigma\rangle \geq 0$ for an isolated system. We have thus directly tested one of the fundamental expressions of the second law of thermodynamics at the level of an isolated quantum system [3].

A comparison of the mean entropy production with the Kullback-Leibler relative entropy between forward and backward states is displayed in Fig. 4(b) as a function of the quench time. We observe good agreement between the two quantities within experimental errors that are due to inhomogeneities in the driving rf field and nonidealities of the field modulation. These results provide a first experimental confirmation of Eq. (1) and the direct verification of the thermodynamic arrow of time in a driven quantum system. They quantify in a precise manner the intuitive notion that the faster a system is driven away from thermal equilibrium (i.e., the bigger the mean entropy production or the shorter the driving time $\tau$ ), the larger the degree of irreversibility, as measured by the relative entropy between a process and its time reversal. 
(a)

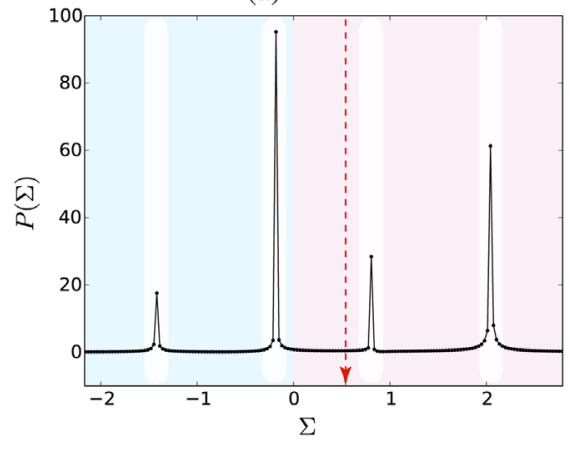

(b)

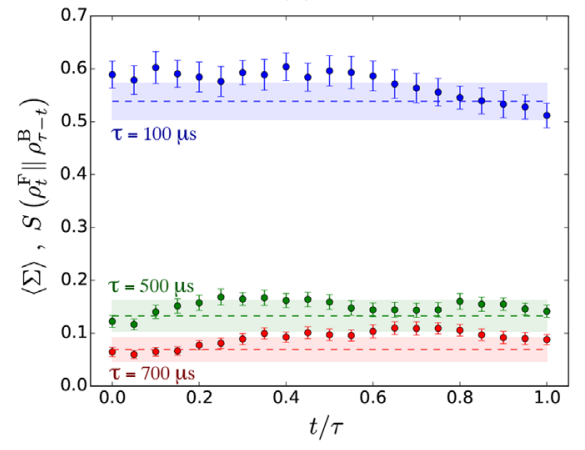

(c)

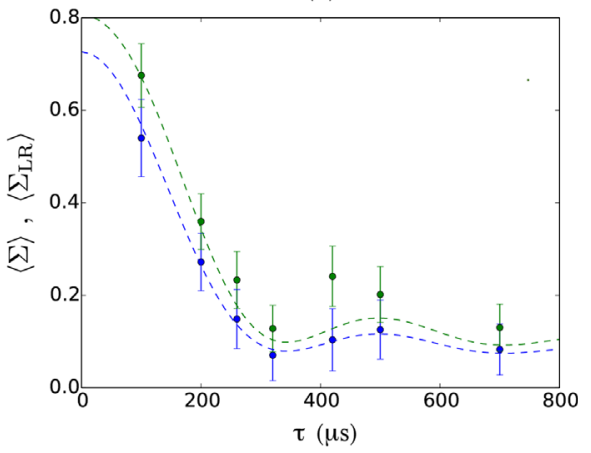

FIG. 4 (color online). (a) Black dots represent the measured negative and positive values of the entropy production $\Sigma$ of the spin-1/2 system after a quench of the transverse magnetic field of duration $\tau=100 \mu \mathrm{s}$. The mean entropy production (red dashed line) is positive, in agreement with the second law. (b) Average entropy production $\langle\Sigma\rangle$ (dashed lines) evaluated through the probability distribution $P(\Sigma)$, and Kullback-Leibler divergence $S\left(\rho_{t}^{F} \| \rho_{\tau-t}^{B}\right)$ between forward and backward states $\rho_{t}^{F}$ and $\rho_{\tau-t}^{B}$ (dots), reconstructed through the tomographic measurements, as a function of time for three different quench durations $\tau=100 \mu \mathrm{s}$ (blue), $500 \mu \mathrm{s}$ (green), and $700 \mu \mathrm{s}$ (red). Good agreement (within experimental uncertainties represented, respectively, by error bars and shadowed regions) between the two quantities is observed, quantifying the arrow of time (1). (c) Mean entropy production $\langle\Sigma\rangle$ (blue dots) and its linear response approximation $\left\langle\Sigma_{\mathrm{LR}}\right\rangle$ (green dots) as a function of the quench time. The difference between the two values, especially for fast quenches (small $\tau$ ), suggests the systematic deviation of the experiment from the linear response regime. The dashed lines represent the results of numerical simulations.

In the linear response regime [3], Onsager derived generic expressions for the entropy production that form the backbone of standard nonequilibrium thermodynamics. These results are, however, limited to systems that are driven close to thermal equilibrium. By contrast, Eq. (1) holds for any driving protocol and thus arbitrarily far from equilibrium. In order to check the general validity of Eq. (1), we use the linear response (LR) approximation of the mean work [17] $\left\langle W_{\mathrm{LR}}\right\rangle=\Delta F+\beta \Delta W^{2} / 2$, where $\Delta W^{2}$ is the variance of the work, to obtain the mean entropy production $\left\langle\Sigma_{\mathrm{LR}}\right\rangle=\beta^{2} \Delta W^{2} / 2$. Figure 4(c) shows the experimental values of $\langle\Sigma\rangle$ and $\left\langle\Sigma_{\mathrm{LR}}\right\rangle$ as a function of the quench duration. We note that the measured irreversible entropy production $\langle\Sigma\rangle$ is close yet systematically distinct from its linear response approximation $\left\langle\Sigma_{\mathrm{LR}}\right\rangle$, the difference being more pronounced for fast quenches (small $\tau$ ), as expected. Figure 4(c) thus suggests that the quenches implemented in the experiment are performed somewhat away from the nonlinear response regime. We also mention that we achieve good agreement between experimental data (dots) and numerical simulations (dashed lines) [29].

Conclusions. - We have assessed the emergence of the arrow of time in a thermodynamically irreversible process by using the tools provided by the framework of nonequilibrium quantum thermodynamics. We have implemented a fast quenched dynamics on an effective qubit in a NMR setting, assessing both the mean entropy produced across the process and the distance between the state of the system and its reverse version, at all times of the evolution. Let us discuss the physical origin of such time asymmetry in a closed quantum system. Using an argument put forward by Loschmidt in the classical context, its time evolution should in principle be fully reversible [1]. How then can a unitary equation, like the Schrödinger equation, lead to Eq. (1) that contains a strictly non-negative relative entropy? The answer to this puzzling question lies in the observation that the description of physical processes requires both equations of motion and initial conditions [1,13]. The choice of an initial thermal equilibrium state singles out a particular value of the entropy, breaks time-reversal invariance, and thus leads to the arrow of time. The dynamics can only be fully reversible for a genuine equilibrium process for which the entropy production vanishes at all times. Moreover, issues linked to the "complexity" of the preparation of the initial state to be used in the forward dynamics (or the corresponding one associated with the time-reversed evolution) have to be considered [33]. By providing an experimental assessment of the microscopic foundation of irreversibility (systematically beyond the linear response regime), our results both elucidate and quantify the physical origin of the arrow of time in the quantum setting of an isolated system.

We thank K. Micadei for valuable discussions. We acknowledge financial support from CNPq, CAPES, FAPERJ, and FAPESP. M.P. is supported by the John Templeton Foundation (Grant ID 43467) and the CNPq "Ciência sem Fronteiras" program through the "Pesquisador Visitante Especial" initiative (Grant No. 401265/2012-9). M.P. and E. L. acknowledge the EU Collaborative Project TherMiQ (Grant Agreement 618074) and the COST Action MP1209. R. M. S. acknowledges the Royal Society and Newton Fund through the 
Newton Advanced Fellowship scheme (Reference No. R1660101). This work was performed as part of the Brazilian National Institute of Science and Technology for Quantum Information (INCT-IQ).

[1] J. L. Lebowitz, Boltzmann's entropy and time's arrow, Phys. Today 46, No. 9, 32 (1993).

[2] H. D. Zeh, The Physical Basis of the Direction of Time (Springer, New York, 2007).

[3] H. B. Callen, Thermodynamics and an Introduction to Thermostatistics (Wiley, New York, 1985).

[4] A.S. Eddington, The Nature of the Physical World (McMillan, London, 1928).

[5] R. M. Velasco, L. S. Garcia-Colin, and F. J. Uribe, Entropy production: Its role in non-equilibrium thermodynamics, Entropy 13, 82 (2011).

[6] F. Schlögl, Stochastic measures in nonequilibrium thermodynamics, Phys. Rep. 62, 267 (1980).

[7] H. Spohn and J. L. Lebowitz, Irreversible thermodynamics for quantum systems weakly coupled to thermal reservoirs, Adv. Chem. Phys. 38, 109 (1978).

[8] S. Deffner and E. Lutz, Generalized Clausius Inequality for Nonequilibrium Quantum Processes, Phys. Rev. Lett. 105, 170402 (2010).

[9] S. Deffner and E. Lutz, Nonequilibrium Entropy Production for Open Quantum Systems, Phys. Rev. Lett. 107, 140404 (2011).

[10] M. Esposito, U. Harbola, and S. Mukamel, Nonequilibrium fluctuations, fluctuation theorems, and counting statistics in quantum systems, Rev. Mod. Phys. 81, 1665 (2009).

[11] M. Campisi, P. Hänggi, and P. Talkner, Quantum fluctuation relations: Foundations and applications, Rev. Mod. Phys. 83, 771 (2011).

[12] M. Campisi and P. Hänggi, Fluctuation, dissipation and the arrow of time, Entropy 13, 2024 (2011).

[13] C. Jarzynski, Equalities and inequalities: Irreversibility and the second law of thermodynamics at the nanoscale, $\mathrm{C}$. Annu. Rev. Condens. Matter Phys. 2, 329 (2011).

[14] R. Kawai, J. M. R. Parrondo, and C. Van den Broeck, Dissipation: The Phase-Space Perspective, Phys. Rev. Lett. 98, 080602 (2007).

[15] S. Vaikuntanathan and C. Jarzynski, Dissipation and lag in irreversible processes, Europhys. Lett. 87, 60005 (2009).

[16] J. M. R. Parrondo, C. Van den Broeck, and R. Kawai, Entropy production and the arrow of time, New J. Phys. 11, 073008 (2009).

[17] J. Liphardt, S. Dumont, S. B. Smith, I. Tinoco, and C. Bustamante, Equilibrium information from nonequilibrium measurements in an experimental test of the Jarzynski equality, Science 296, 1832 (2002).

[18] V. Blickle, T. Speck, L. Helden, U. Seifert, and C. Bechinger, Thermodynamics of a Colloidal Particle in a
Time-Dependent Nonharmonic Potential, Phys. Rev. Lett. 96, 070603 (2006).

[19] J. Gieseler, R. Quidant, C. Dellago, and L. Novotny, Dynamic relaxation of a levitated nanoparticle from a non-equilibrium steady state, Nat. Nanotechnol. 9, 358 (2014).

[20] U. Seifert, Stochastic thermodynamics, fluctuation theorems and molecular machines, Rep. Prog. Phys. 75, 126001 (2012).

[21] S. Ciliberto, R. Gomez-Solano, and A. Petrosyan, Fluctuations, linear response, and currents in out-ofequilibrium systems, Annu. Rev. Condens. Matter Phys. 4, 235 (2013).

[22] D. Andrieux, P. Gaspard, S. Ciliberto, N. Garnier, S. Joubaud, and A. Petrosyan, Entropy Production and Time Asymmetry in Nonequilibrium Fluctuations, Phys. Rev. Lett. 98, 150601 (2007).

[23] I. S. Oliveira, T. J. Bonagamba, R. S. Sarthour, J. C. C. Freitas, and R. R. deAzevedo, NMR Quantum Information Processing (Elsevier, New York, 2007).

[24] L. M. K. Vandersypen and I. L. Chuang, NMR techniques for quantum control and computation, Rev. Mod. Phys. 76, 1037 (2005).

[25] D. G. Cory, A. F. Fahmy, and T. F. Havel, Ensemble quantum computing by NMR spectroscopy, Proc. Natl. Acad. Sci. U.S.A. 94, 1634 (1997).

[26] T. B. Batalhão, A. M. Souza, L. Mazzola, R. Auccaise, R. S. Sarthour, I. S. Oliveira, J. Goold, G. De Chiara, M. Paternostro, and R. M. Serra, Experimental Reconstruction of Work Distribution and Verification of Fluctuation Relations at the Full Quantum Level, Phys. Rev. Lett. 113, 140601 (2014).

[27] R. Dorner, S. R. Clark, L. Heaney, R. Fazio, J. Goold, and V. Vedral, Extracting Quantum Work Statistics and Fluctuation Theorems by Single Qubit Interferometry, Phys. Rev. Lett. 110, 230601 (2013).

[28] L. Mazzola, G. De Chiara, and M. Paternostro, Measuring the Characteristic Function of the Work Distribution, Phys. Rev. Lett. 110, 230602 (2013).

[29] See Supplemental Material at http://link.aps.org/ supplemental/10.1103/PhysRevLett.115.190601 for additional experimental details and theoretical analysis.

[30] J. Kurchan, A quantum fluctuation theorem, arXiv:condmat/0007360; H. Tasaki and C. Jarzynski, Relations for quantum systems and some applications, arXiv:cond-mat/ 0009244v2.

[31] G. E. Crooks, Entropy production fluctuation theorem and the nonequilibrium work relation for free-energy differences, Phys. Rev. E 60, 2721 (1999).

[32] P. Talkner and P. Hänggi, The Tasaki-Crooks quantum fluctuation theorem, J. Phys. A 40, F569 (2007).

[33] G. B. Lesovik, On the law of increasing entropy and the cause of the dynamics irreversibility of quantum systems, JETP Lett. 98, 184 (2013). 